\title{
Cost analysis of two different configurations for a distribution network design
}

\begin{abstract}
One significant decision in supply chain management is to determine the configuration of the supply chain network. However, studies that analyze different configurations are rare in the literature. This paper considers a supply chain network consisting of one supplier and a number of retailers. Two different configurations were proposed and were compared in terms of total cost for the network. In the first configuration, retailers directly order to the supplier and hold the inventory to meet the demand of customers. However, in the second configuration, a number of intermediate facilities were established to order products from the supplier and satisfy the demands of retailers. The inventory of retailers in the latter configuration is stored in the intermediate facilities. These two configurations were compared in terms of total cost. Numerical calculations indicate that the latter configuration was preferred in most cases.
\end{abstract}

Keyword: Distribution network design; Facility location; Inventory control; Network configuration; Supply chain management 